\title{
OPTICAL OBSERVATIONS OF NONTHERMAL GALACTIC RADIO SOURCES
}

\author{
R. MINKOWSKI \\ Mount Wilson and Palomar Observatories, Pasadena, California, U.S.A.
}

A total of 9 nonthermal sources near the plane of the Galaxy have now been definitely identified with nebulosities. The identifications are well established; positions and sizes of the nebulosities and of the sources are in satisfactory agreement. At least three types of objects can be recognized:

1. Remnants of supernovae of type I.

2. The Cassiopeia A source, a remnant of a supernova of type II.

3. The Cygnus loop and several other large nebulosities which are most likely of similar origin. They also may be remnants of supernovae type II.

The large size and the low surface brightness of these objects render their detailed investigation a difficult and time-consuming task. The true picture may emerge slowly, and conclusions drawn at an early stage of the work may have to be discarded as the investigation progresses.

\section{THE REMNANTS OF THE SUPERNOVAE OF TYPE I}

Unlike the Crab nebula (the remnant of the supernova of 1054, which has long been known and investigated in great detail), the remnant of Tycho's nova of 1572 and that of Kepler's nova of 1604 , both undoubtedly supernovae of type I, are very inconspicuous and difficult objects.

The remnant of Kepler's nova [1] consists of a relatively conspicuous fanshaped mass of filaments and several faint wisps of nebulosity. These details surround Kepler's position of the nova (Fig. 1), and show small motions away from the center of the area. Measurements of radial velocities within the fan-shaped mass, which precedes Kepler's position, and within the group of faint filaments south following show that the mean velocities of both details are very nearly equal, $-230 \mathrm{~km} / \mathrm{second}$ and $-220 \mathrm{~km} / \mathrm{second}$ respectively, with a scatter of the order $\pm 50 \mathrm{~km} / \mathrm{second}$ within each mass. If the velocity of the expansion is equal to that of the Crab nebula, $1200 \mathrm{~km} / \mathrm{second}$, then both details must be near the edge of the nebulosity. After the motions have been measured and more radial velocities have been observed it may be possible to arrive at a definite description and a reliable position. It seems probable that the position is slightly north following Kepler's position, but well within its uncertainty of \pm 1 minute of arc. The nebulosity may be elliptical, with a major axis of about 2'.5 near position angle 55 degrees. The position of the remnant will in any case be in satisfactory agreement with the positions found for the source $[2,3,4]$.

The size of the remnant may be compatible with the diameter to half- 
intensity points of $<1$ minute of arc for a gaussian source found by Baldwin and Edge [4]. It does not seem possible to maintain the conclusion of Rishbeth and Little [5] that the source was partially occulted by the moon and that its angular diameter is 10 minutes of arc.

If the velocity of expansion is equal to that of the Crab nebula, the distance of Kepler's nova must be about equal to that of the Crab, 1000 parsecs, since the sizes are nearly in the same ratio as the times elapsed since the outbursts. If Kepler's nova was of average absolute magnitude, the visual absorption in front of the nova must be about 4 magnitudes, a conclusion in agreement with the low surface brightness of the remnant [1].

The remnant of Tycho's nova is even less conspicuous than that of Kepler's nova. A long filament, an extremely faint arc of nebulosity,

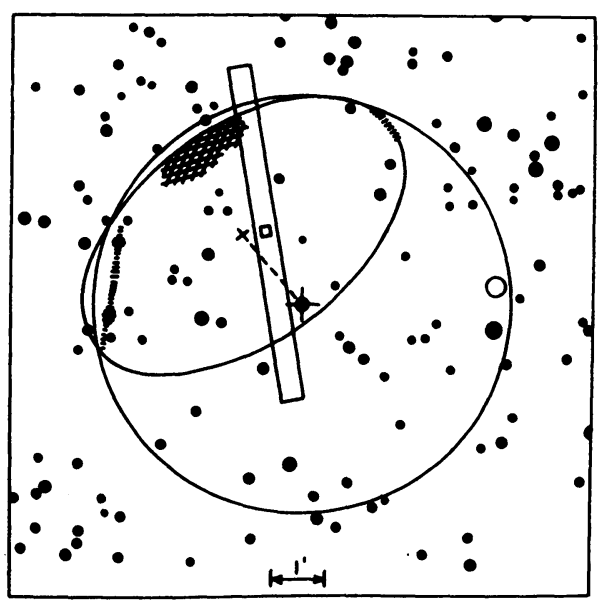

Fig. 2. B Cassiopeiae. The open circle marks the position from Tycho's observations. The upright and oblique crosses mark the centers of the smallest circle and ellipse respectively that can be fitted to the visible fragments. The square marks the position of the radio source by Baldwin and Edge; the rectangle its mean error.

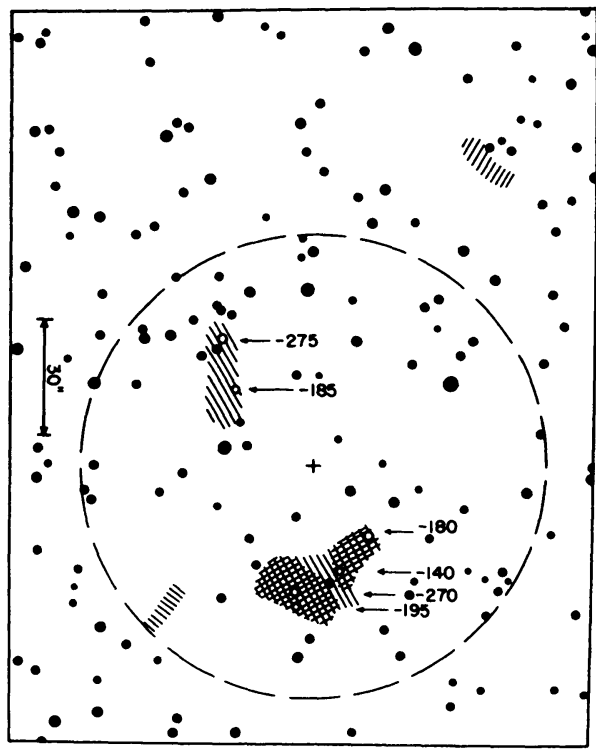

FIg. 1. Nova Ophiuchi of 1604 . The position of the nova is marked by a cross; the circle indicates the mean error of the computed position.

and a short filament (Fig. 2) seem to define part of the outline of the remnant. The filaments show motions toward the outside. The radial velocity of the long filament is near zero; it cannot be measured accurately because $\mathrm{H} \alpha$, the only observable line, is blended with an extremely faint $\mathrm{H} \alpha$ emission that seems to cover the whole field. The low radial velocity proves that the long filament is very close to the edge. It will be possible to determine a reliable position of the center of the nebulosity after the motions of the filaments have been measured. It seems possible that the position will be near the line connecting the centers of the smallest circle and of the smallest ellipse that can be fitted to the visible details. It seems definite that the position of the source determined by Baldwin and 
Edge [4] will be in close agreement with that of the remnant. The position following from Tycho's observations [6] agrees well in declination, but deviates by about $40^{\mathrm{s}}$ in right ascension from the position of the remnant. No explanation can be offered for the unexpectedly large error of Tycho's position; the unfavorable distribution of his comparison stars may be its cause.

The remnant has a diameter or, if it is elliptical, a major axis of about 7 minutes of arc about in position angle 130 degrees. This is in good agreement with the diameter of 5.4 between half-intensity points for a gaussian source found by Baldwin and Edge [4]. If the velocity of expansion is the same as in the Crab nebula, the distance is 360 parsecs. If the supernova was of average absolute magnitude, the visual absorption covering the field must be about 4 magnitudes, easily explaining the faintness of the remnant.

\section{THE CASSIOPEIA A SOURCE}

The continued investigation of the nebulosity coinciding with the Cassiopeia A source has now reached a stage in which the true nature of the object emerges clearly [7]. Certain conclusions formed at earlier phases of the investigation [8] have become untenable. There are good reasons to believe that the following description of the object is final, except for details of minor importance.

Better photographs in red light show the object as a circular nebulosity with a radius of 2 minutes of arc. A flare extends to a distance of about 3'.8 from the center of the circle in position angle 70 degrees. The position of the center of the circular area (Table I) seems to be well defined; the agreement with the radio position by F. G. Smith [9] remains good, particularly in view of the size and irregular shape of the nebulosity as a whole.

TABLE I

Positions of Cassiopeia A

\begin{tabular}{lll} 
& \multicolumn{1}{c}{$\alpha(1950)$} & $\delta(1950)$ \\
Center of circular area & $23^{\mathrm{h}} 21^{\mathrm{m} 9 \mathrm{~s}} 4$ & $+58^{\circ} 32^{\prime} 27^{\prime \prime}$ \\
Center of expansion & $23^{\mathrm{h}} 21^{\mathrm{m}} 11 \mathrm{~s} .8 \pm 0.4$ & $+58^{\circ} 32^{\prime} 16^{\prime \prime} \pm 8^{\prime \prime}$ \\
Position of source* & $23^{\mathrm{h}} 21^{\mathrm{m}} 12^{\mathrm{s}} .0 \pm 1^{\mathrm{s}}$ & $+58^{\circ} 32{ }^{\prime} 1 \pm 0.7$
\end{tabular}

* F. G. Smith, Nature, 168, 555, 1951.

The nebulosity contains more than 200 small condensations. The existence of two drastically different kinds of condensations is now still more definitely established. Sharp broken bits, which can be photographed only in the red, have small radial velocities, and show even with an interval of 6 years no trace of transverse motions. About twenty such semi-stationary condensations have been noted. The majority of the features are more diffuse fast-moving condensations which can be seen both in the blue and the red.

The motions of the fast-moving condensations show clearly the picture of an expanding object [8]. All measured points belong to the circular area; no point belonging to the flare was measurable. The center of expansion, derived from a least square solution (Table I), agrees satisfactorily with the 


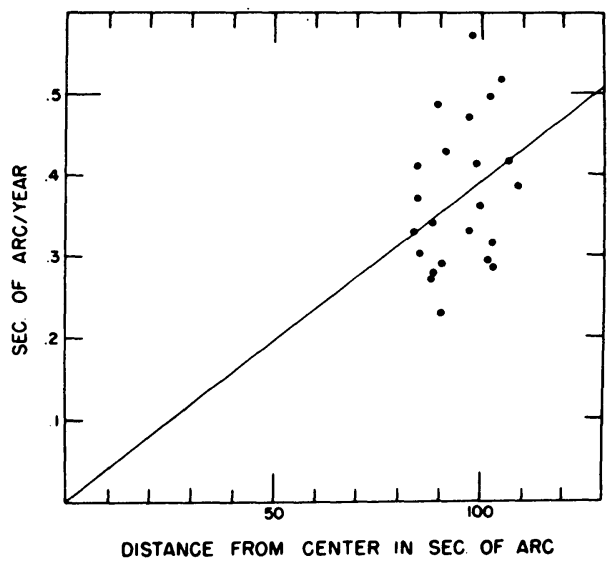

Fig. 3. Proper motions of fast-moving condensations in Cassiopeia A as functions of distance from the center. center of the circular area, but its position is less reliable. The position of the center of the circular area will therefore be used as center for all discussions of motions and radial velocities.

The absolute values of the motions are shown in Fig. 3 as a function of the distance from the center. The scatter around the straight line that would correspond to a simple expansion is large, but the increase of motion with distance is definite. The mean expansion is given by $s / r=$ $0.390 \pm 0.019$, in which $s$ is in seconds of arc per year and $r$ in seconds of arc. If the expansion was uniform, it started $256 \pm 14$ years ago.

The radial velocities as a function of the distance from the center are shown in Fig. 4, on the left for the fast-moving condensations, on the right for the semi-stationary ones. It seems clear that the velocities of condensations belonging to the flare have to be disregarded in a discussion of the over-all pattern. If this is done, the fast-moving condensations quite clearly show the picture of an expanding nebulosity. The velocities of approach are

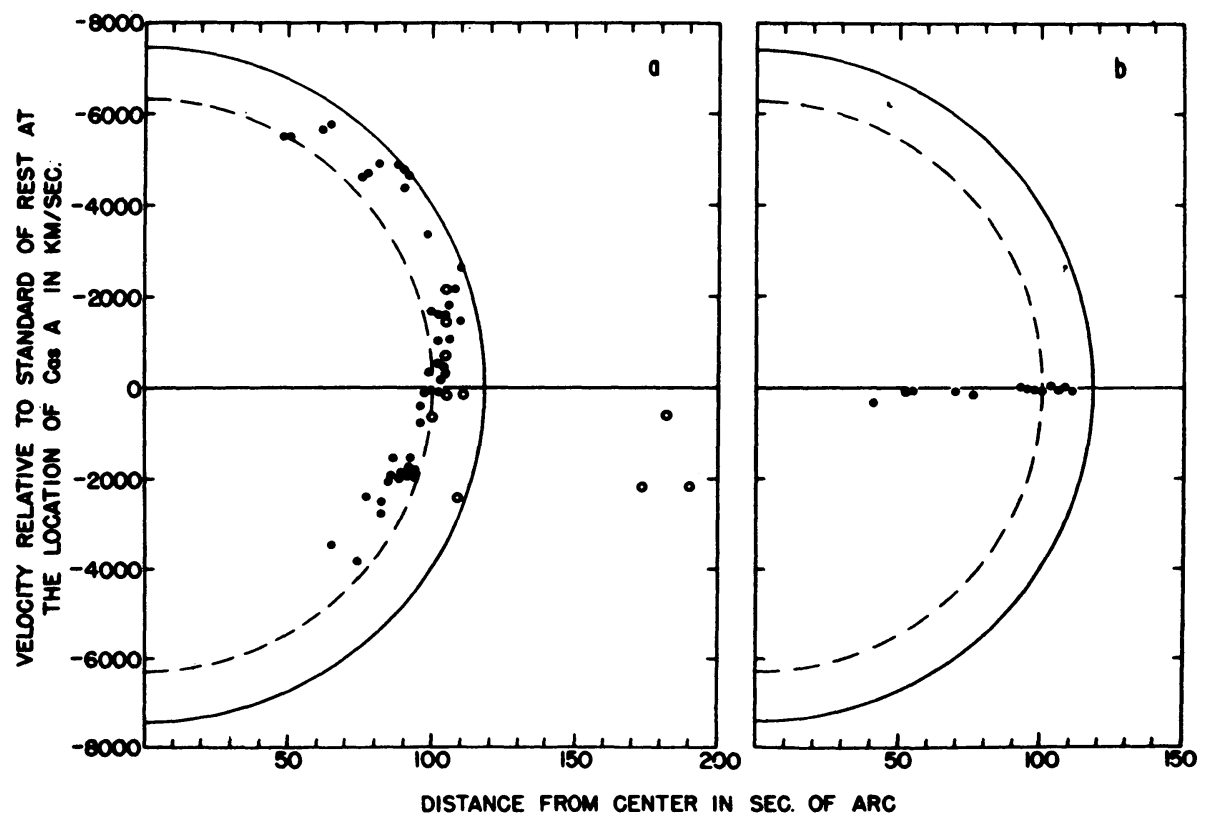

FIG. 4. Radial velocities in Cassiopeia $\mathrm{A}$ as function of the distance from the center. 
obviously smaller than the velocities of recession. The interpretation which suggests itself is that the front half of the expanding mass has been decelerated more than the rear half. The closest ellipse that can be fitted to the receding side corresponds to a velocity of expansion of $7440 \mathrm{~km} / \mathrm{second}$ and a radius of 118 seconds of arc. The thickness of the expanding shell as indicated by the range of point scatter on the receding side is 18 seconds of arc. On the approaching side, the velocity of expansion of the outer edge is $6000 \mathrm{~km} /$ second.

The velocity of expansion of $7440 \mathrm{~km} / \mathrm{second}$ in combination with the observed motion leads to a distance of 3400 parsecs, with an estimated uncertainty of perhaps 300 parsecs. This value is in excellent agreement with the results derived from the study of the $21 \mathrm{~cm}$ absorption lines [10,11]. At a distance of 3400 parsecs, Cassiopeia A is 140 parsecs south of the galactic plane in a region where Westerhout [12] finds a density of hydrogen atoms of $0.2 \mathrm{~cm}^{-3}$. The linear diameter of the circular nebulosity is 4.0 parsecs.

There can be no doubt that the earlier attempt to derive the distance from the apparent random components of radial velocities and motions has led to a result which cannot be correct. The reason for the failure of that approach is to be sought in a misinterpretation of the apparent random motions. In addition to errors of measurement they must represent almost entirely the effects of variability of the fast-moving condensations as to shape and to arrangement of overlapping parts. The true random motions must be small; they may amount to about 15 per cent of the apparent random motions.

As is to be expected from the different colors of the fast-moving and of the semi-stationary condensations, the spectra of these two types of condensations are radically different. The interpretation of the observed spectra [8] remains essentially unchanged by the improved values of collision crosssections.

Now that the distance of the nebulosity is safely established, the linear size of the condensations and their mass can be stated. Most of the average condensations of both types have apparent diameters of 1 to 2 seconds of arc. With a distance of 3400 parsecs, their linear diameters are 5 to $10 \times 10^{16}$ $\mathrm{cm}$. The mass of the average fast-moving condensation is then about $2 \times 10^{30}$, and the combined mass of the roughly 200 fast-moving condensations thus is about $2 \times 10^{32}$ grams. Only a fraction of the total shell is visible, however, and the total mass of the complete moving shell would probably be close to 1 solar mass. The average mass of a semistationary condensation is about $10^{32}$ grams, and the combined mass of the relatively few condensations of this type also amounts to about 1 solar mass, but it seems plausible to assume that this mass was not part of the moving shell, but was gathered in the formation of the semistationary condensations.

There is no longer doubt that the nebulosity is the remnant of a type II supernova. This is the only type of object for which velocities of expansion of the order $5000 \mathrm{~km} / \mathrm{second}$ have been reported; a value of $7500 \mathrm{~km} / \mathrm{second}$ is well within the range of possible interpretations. Moreover, no other type of nova or supernova is known to have an ejected mass of the order of 1 solar mass. An ejected mass of this order seems acceptable for the type II 
supernovae, which as members of Baade's Population I may be very massive stars. At a distance of 3400 parsecs and with an interstellar absorption of about 6 magnitudes over the center of the nebulosity, the expected apparent brightness of a supernova of type II is about +5 magnitudes, too faint to expect discovery of the supernova.

The question may be raised whether the larger deceleration of the front side compared to the rear side of the shell is consistent with reasonable densities of the interstellar medium. If by neglecting all possible energy losses we assume that the shell is decelerated merely by sweeping up interstellar material, we find that at the average interstellar density $n_{H}$ of $0.2 \mathrm{~cm}^{-3}$ a shell of 1 solar mass would have lost only about 10 per cent of its initial velocity when its radius has become 2 parsecs. To explain the observed reduction of the frontside's velocity compared to the backside's the approaching front of the shell must have encountered a denser cloud with $n_{H}$ of 0.8 $\mathrm{cm}^{-3}$ while the receding back has moved through material of average density.

The mass of a semi-stationary condensation is about 50 times that of the average fast-moving condensation. This is about the inverse ratio of the velocities. It is thus permissible to think that the semi-stationary condensations have been formed by fast-moving condensations encountering very dense material. Since the velocities of the semi-stationary condensations nearer the center are negative, one has to assume that they have been formed from the approaching front of the shell, which is also the most strongly retarded part. Some considerations, such as the fact that there are no condensations of an intermediate type, suggest that the formation of a semi-stationary condensation is a rather sudden process requiring interstellar material of high density of the order $10^{5}$ to $10^{6} \mathrm{~cm}^{-3}$ in small condensations.

\section{THE CYGNUS LOOP AND RELATED OBJECTS}

The Cygnus loop, a slowly expanding nebulosity whose brightest parts are NGC 6960 and NGC 6992, is the only example of a small group of objects that has been investigated in some detail [13]. IC 443 is certainly very similar. Other objects belonging to this group are the sources HB 9 (IAU 04N4A) and HB 21. The map of flux densities for the Taurus region by Seeger, Westerhout, and van de Hulst [14] gives some suggestion that the large filamentary nebulosity S 147 [15] may be a source also belonging to this group. Puppis A, in which only low radial velocities have been observed, is probably best included in this group.

The average picture shown by the radial velocities in the Cygnus loop is of an incomplete expanding shell whose inner boundary has a diameter of about 80 minutes of arc and a velocity of expansion of $65 \mathrm{~km} / \mathrm{second}$. The diameter of the outer boundary is 170 minutes of arc and it has a velocity of expansion of $115 \mathrm{~km} / \mathrm{second}$. In the south, an extension reaches to a distance of 145 minutes of arc from the center. From the velocity of expansion at the outer border and Hubble's value of the motion of 0.03 seconds of arc per year, a distance of 770 parsecs is obtained The diameter of the main part of the nebula is then 40 parsecs. 
IC 443 resembles the Cygnus loop in many ways, except that the motions are smaller. It seems certain that it is a very similar object, but at a larger distance, perhaps 2000 parsecs or even more.

The brightest filaments of the Cygnus loop and of IC 443 have electron densities of $500 \mathrm{~cm}^{-3}$ [16]. With a mean electron density of $200 \mathrm{~cm}^{-3}$, the mass of a typical bright filament with a length of 1 parsec and a diameter of $7 \times 10^{-3}$ parsecs becomes about $2 \times 10^{-4}$ solar masses. Since there are hundreds of filaments and many more diffuse details, the mass of the visible nebulosity must be of the order of 0.1 solar masses. Since the shell is quite incomplete, it is certain that the total mass, large parts of which may have cooled and may now be dark, is very much larger.

Spectroscopic investigations leave now no doubt that the excitation of emission in the Cygnus loop is collisional. This supports strongly the suggestion by Oort that the Cygnus loop is a shell originally ejected with high velocity and decelerated by collision with interstellar clouds. Under this assumption the present mass of the shell must be mainly the mass of the interstellar material swept up by the shell, or $7 \times 10^{2} n_{H}$ solar masses for a shell of 40 parsecs diameter. With a velocity of expansion of $100 \mathrm{~km} / \mathrm{second}$, the momentum is $7 \times 10^{4} n_{H}$ (solar masses $\times \mathrm{km} / \mathrm{second}$ ). If $n_{\boldsymbol{H}}$ is of the order of $10^{-2} \mathrm{~cm}^{-3}$ or larger, this momentum cannot be provided by ordinary novae or supernovae of type I. A supernova of type II, with a momentum of $7.5 \times 10^{3}$, such as that whose remnant is Cassiopeia $\mathrm{A}$, would require the very plausible value of $0.1 \mathrm{~cm}^{-3}$ for $n_{H}$. The appearance of the Cygnus loop and of IC 443 as incomplete thick shells may result if, as a result of density inhomogeneities in the interstellar medium, matter ejected in different directions has undergone different retardations. To explain the observed appearance, it is necessary only that in some directions interstellar densities to 1.2 $\mathrm{cm}^{-3}$ have been encountered by the expanding shell.

This interpretation may lead to an explanation of the curious fact found by Rishbeth [17] that in IC 443 the nonthermal radio source resembles in the distribution of surface brightness the nebula whose line emission seems to be of thermal origin. If at this moment the matter at the northeast edge of the nebula encounters interstellar matter of particularly high density, strong heating may lead to strong optical emission, and compression of matter and magnetic fields may lead to strong nonthermal radio emission.

The assumption that objects like the Cygnus loop are strongly deceleratedexpanding shells leads to the expectation that their size distribution would show considerable preference for large diameters since the number of objects of a given size will be inversely proportional to the velocity of expansion reached at that size. This may explain the curious fact that only two or three objects of moderate size are known that might possibly belong to this class of objects; none of these objects has been observed as a radio source.

If the Cygnus loop were the result of uniform expansion, its age would be 150,000 years, an upper limit to the true age. If it is the result of decelerated expansion of a shell with the initial momentum $7 \times 10^{3}$ [solar masses $\times$ $\mathrm{km} / \mathrm{second]}$ in interstellar material of $10^{-1} \mathrm{H}$ atoms per $\mathrm{cm}^{3}$, its age is 50,000 years. The age at which such an object disappears because the velocity of 
expansion has decreased to the level of random velocities may be of the order of 100,000 years. Since type II supernovae seem to occur with a frequency of one supernova per galaxy per 50 years, one would expect that the number of objects of this type in the Galaxy is of the order 2000. This number seems rather large compared to 5 or perhaps 8 known objects, particularly since the frequency of members of Population I should not increase strongly in the central parts of the galaxy. But many such objects may be hidden by obscuration, the average lifetime might be shorter, and the frequency of supernovae of type II in the galaxy is not reliably known. In view of the crudity of the discussion and the lack of basic data, the discordance does not appear serious at this time. Thus, it seems to be at least a reasonable working hypothesis that such objects as the Cygnus loop are remnants of supernovae of type II.

\section{REFERENCES}

[1] Baade, W. Ap. J. 97, 119, 1943.

[2] Shakeshaft, J. B., Ryle, M., Baldwin, J. E., Elsmore, B., and Thomson, J. H. Mem. R.A.S. 67, 106, 1955.

[3] Mills, B. Y., Little, A. G., and Sheridan, K. V. Aust. J. Phys. 9, 84, 1956.

[4] Baldwin, J. E., and Edge, D. O. The Observatory, 77, 139, 1957.

[5] Rishbeth, H., and Little, A. G. The Observatory, 77, 71, 1957.

[6] Baade, W. Ap. J., 102, 309, 1945.

[7] A detailed discussion will be in a forthcoming paper by W. Baade and R. Minkowski.

[8] Minkowski, R. Radio Astronomy (I.A.U. Symposium No. 4, 1955). Cambridge, England, 1957, p. 114.

[9] Smith, F. G. Nature, 168, 555, 1951.

[10] Hagen, J. P., Lilley, A. E., and McClain, E. F. Ap. J. 122, 361, 1955. Radio Astronomy (I.A.U. Symposium No. 4), p. 82.

[11] Muller, C. A. Ap. J. 125, 830, 1957.

[12] Westerhout, G. B.A.N. 13, 201, 1957.

[13] Minkowski, R. Rev. Mod. Phys. 30, 1048, 1958.

[14] Seeger, Ch. L., Westerhout, G., and van de Hulst, H. C. B.A.N. 13, 89, 1956.

[15] Gaze, V. F., and Shain, G. A. Izv. Crimean Astrophys. Obs. 7, 19, 1951; 9, 52, 1952.

[16] Osterbrock, D. E. P.A.S.P. 70, 180, 1958.

[17] Rishbeth, H. Aust. J. Phy8. 9, 494, 1956.

\section{Discussion}

Oort: In estimating the interstellar density from the deceleration of the near side of the Cassiopeia $\mathrm{A}$ shell do you assume that it was a complete shell?

Minkowski: Yes. 